\title{
Microbiome in Chronic Lung Diseases
}

\author{
Manoj J. Mammen, $M D^{1,2}$, and Sanjay Sethi, $M D^{1,3}$
}

${ }^{1}$ Division of Pulmonary, Critical Care, and Sleep Medicine and ${ }^{2}$ Department of Biomedical Informatics, Jacobs School of Medicine, University at Buffalo, State University of New York, NY, USA; ${ }^{3}$ Veterans Affairs Western New York Healthcare System, Buffalo, NY, USA

\section{ABSTRACT}

Newer genetic sequencing methods confirm the presence of a lung microbiome in healthy individuals. Microbiome imbalances can contribute to disease as they disrupt normal micro-environmental stimuli for the human host. Recent studies in chronic lung disease suggest that imbalances of the lung microbiome are associated with disease pathogenesis. The most common changes in the microbiome in chronic lung diseases are a decrease in diversity and the predominance by Proteobacteria. Additional changes in the lung microbiome have been described in various diseases when the clinical state changes from stable lung disease to respiratory exacerbation. Understanding the impact of the microbiome with robust and innovative tools on chronic lung disease pathogenesis, progression and exacerbation may ultimately lead to microbiome directed therapies. (BRN Rev. 2017;3:102-20) Corresponding author: Sanjay Sethi, ssethi@buffalo.edu

Key words: Asthma. Chronic obstructive pulmonary disease. Cystic fibrosis. Metagenome. Microbiota. 


\section{INTRODUCTION}

The advent of newer technologies enables exploration of the human microbiome. The human microbiome or microbiota is the collection of microorganisms that live in and on the human body ${ }^{1}$. In an individual, microbial cells outnumber human cells by a factor of 100 in some estimates, but recent estimates conclude an equal number of microbial and human cells ${ }^{2}$, with microbial genes outnumbering human genes by a factor of $100^{3}$. This lopsided ratio has important ramifications, including supporting the hologenome theory which asserts the human microbiome likely co-evolved with its human hosts, as there is evidence that it provides metabolic pathways that human cells do not have $\mathrm{e}^{4}$. The complex amalgamation of microbiota in human microenvironments provide for symbiotic interactions necessary for human health ${ }^{5}$. Dysbiosis, or the disruption of the microbiome and its related collection of genes, or metagenome can lead to disease since the metagenome is a unique juxtaposition of genomics and environment, providing both unique metabolic functions and micro-environmental stimuli for the human host $^{6}$. The human microbiome project (HMP) undertaken in $2007^{7}$ was initially designed to understand the "normal" microbiome across the human lifespan, and to understand the significance of the microbiome in human health and disease. Subsequently, studies have been investigating the various human microbiota in disease.

\section{THE HUMAN MICROBIOME CAN BE READILY CHARACTERIZED WITH NEW GENETIC SEQUENCING TECHNOLOGY}

The advent of massive parallel sequencing of the polymerase chain reaction (PCR) products or amplicons enables high throughput determination of the microbial ecology in a biological sample and categorization of metagenomes in a human habitat ${ }^{8}$. It is now known that less than $1 \%$ of bacteria collected from soil that are seen under the microscope form colonies on culture, and only $50 \%$ of bacteria sampled from human sites identified by $16 \mathrm{~S}$ ribosomal ribonucleic nucleic acid (RNA or rRNA) sequencing techniques are recovered by traditional culture techniques ${ }^{9,10}$. A common method to characterize microbiome is sequencing deoxyribonucleic acid (DNA) of the RNA component of the 16S rRNA gene, a highly conserved bacterial gene ${ }^{11}$. It has conserved sequences to allow cloning with specific PCR primers ${ }^{12}$, but sequencing several of the nine hyper-variable regions of the RNA component of the 16S rRNA gene, designated V1 to V9 allows identification of bacteria at the genus level (16S rRNA gene sequencing) ${ }^{12}$. Not one hypervariable region can differentiate amongst all bacteria, however the $\mathrm{V} 1-\mathrm{V} 3$ region distinguishes nearly all bacteria considered pathogenic at the genus level, except for the Enterobacteriaceae genus ${ }^{13}$. An advantage of the genetic sequencing methods as opposed to traditional culture methods is the avoidance of over-representation of easily cultured bacteria over bacteria that is difficult to culture. A recent microbiome study reveals that Haemophilus and Moraxella genera are detected more frequently with the novel genetic techniques versus traditional culture methods, while the presence of Enterobacteriaceae is overestimated since it is easier to detect Enterobacteriaceae versus Haemophilus and Moraxella by conventional culture ${ }^{14}$. To classify different taxa of bacteria by $16 \mathrm{~S}$ rRNA gene sequence data, the similarity of the sample sequences is compared to a known taxonomic data set, using a $97 \%$ similarity threshold, sequences are then grouped into operational taxonomic units (OTU), since 
the bacterial species associated with a specific genetic sequence may not be known ${ }^{4}$.

16S rRNA gene sequencing provides descriptive assessment of taxonomic compositions and phylogenetic diversity of microbiomes, but does not distinguish the metabolic capabilities of the microbiome. Whole genome sequencing of the microbiome or metagenomic sequencing identifies the genes which underlie the collective metabolic and functional capacity of a microbiome ${ }^{15}$. Metagenomic sequencing had entailed time-consuming, costly and data-intensive sequencing (via Sanger sequencing method) of the entire set of genes in microbiome habitat. Though this has been simplified with the use of massively parallelized sequencing, it still involves complicated post-sequencing methods to avoid analysis of human DNA in addition to microbial DNA ${ }^{16}$. Recently developed bioinformatics software can infer the metabolic pathways of a metagenome given the sample's taxonomic composition ${ }^{17}$.

After a microbiome's taxonomic composition is detailed, the characteristic parameters can be determined. These include the number of unique taxonomic units detected in a sample (community richness), the relative numbers of taxonomic units in a sample (evenness), the diversity of a sample with either a measure of the richness and evenness within a sample (alpha diversity, e.g. Shannon Index), and/or the similarity of the bacterial composition between samples, (beta diversity, e.g. Brays-Curtis or UniFrac) ${ }^{18}$ (Table 1).

\section{THERE IS A LUNG MICROBIOME IN HEALTHY INDIVIDUALS}

It was believed that the lungs are sterile in healthy humans ${ }^{19}$ and the lungs were excluded in initial HMP studies. However, the belief was based on data using traditional culture techniques. The first report, by Hilty et al. ${ }^{20}$, utilizing genetic sequencing techniques, revealed a lower airways microbiome in healthy humans, compared the microbiome of three cohorts with either asthma, chronic obstructive pulmonary disease (COPD) or healthy controls. The microbiome of nasal cavity and oropharynx sampled by surface swabs and that of the lower airways sampled via bronchial brushing of the mucosal surface of the left upper lung lobe were compared in the study. Sequencing of the microbiome in the bronchial brushings of the control subjects $(\mathrm{n}=8)$, identified multiple bacterial phyla. Additionally, bacterial abundance in the range of 1-10 million bacterial cells/sample was similar in the three groups, but there were significant differences in microbiome composition amongst the groups.

An abundant microbiome in the healthy lung was met initially with disbelief ${ }^{21}$. One concern was that the lower airway microbiome results could be confounded by upper airway contamination, since the oropharynx has a bacterial load several orders of magnitude higher than that of the lower airways. Furthermore, even sterilized reagents and fluids used in the sampling and processing procedures could contain DNA from dead bacteria detectable with $16 \mathrm{~S}$ rRNA gene sequencing. Adequate negative controls are required in order to control and account for such environmental contamination, which were not performed in this study. The next published study by Erb-Downward et al. ${ }^{22}$ also showed a similarly abundant microbiome in healthy human airways sampled by bronchoalveolar lavage (BAL); however, the same limitations applied to this study. 
TABLE 1. Definitions of commonly used terms in study of the lung microbiome

\begin{tabular}{|c|c|}
\hline Terms & Description \\
\hline $16 S$ ribosomal gene & Gene encoding a $16 \mathrm{~S}$ subunit of bacterial ribosomes that is highly conserved between different bacterial species \\
\hline 16S rRNA gene sequencing & $\begin{array}{l}\text { Determining the DNA sequence of a } 16 \mathrm{~S} \text { ribosomal gene, particularly the regions that are variable amongst } \\
\text { species }\end{array}$ \\
\hline Alpha diversity & A measure of the richness and evenness within a sample, (e.g. Shannon Index) \\
\hline Beta diversity & Represents the similarity of the bacterial composition between samples, (e.g. Brays-Curtis or UniFrac) \\
\hline Bioinformatics & Multidisciplinary field pertaining to the study, development and use of tools to manipulate biological data \\
\hline DNA microarray & Two-dimensional array of specific DNA sequence probes to hybridize complementary sequences \\
\hline Double bronchoscope method & $\begin{array}{l}\text { Sampling technique, whereby one bronchoscope anesthetized the airway, and a second bronchoscope with } \\
\text { an unused and uncontaminated channel is used to obtain lower airway samples via bronchial brushings } \\
\text { and/or lavage }\end{array}$ \\
\hline Dysbiosis & Disruption of the microbiome and its related collection of genes \\
\hline Evenness & Relative numbers of taxonomic units in a sample \\
\hline Hologenome theory & Theory asserting human microbiome co-evolved with its host \\
\hline Human microbiome project & $\begin{array}{l}\text { National Institutes of Health funded project to comprehensively characterize of the human microbiome and its role } \\
\text { in human health and disease }\end{array}$ \\
\hline Island biogeography theory & $\begin{array}{l}\text { Theory asserting different human anatomic locations constitute different "islands" of habitation with temporal } \\
\text { and spatial variation }\end{array}$ \\
\hline Metagenome & The genes and their expression products associated with microorganisms from a habitat \\
\hline Microbiome & The collection of all the microorganisms living in a habitat \\
\hline Microbiota & The collection of all microorganisms living in association with the human body \\
\hline Next generation sequencing & $\begin{array}{l}\text { High throughput technologies, which allow rapid sequencing of DNA in parallel as opposed to the traditional DNA } \\
\text { sequencing approach, the Sanger method. (i.e. Roche } 454 \text { Pyrosequencing, Illumina HiSeq, Illumina MiSeq) }\end{array}$ \\
\hline Operational taxonomic unit & Categorization of organisms into species or groups of species by DNA sequence data \\
\hline Phylogenetics & The study of the evolutionary history of an organism \\
\hline Richness & The number of unique taxonomic units detected in a sample \\
\hline Taxonomy & Classification of organisms \\
\hline Topological continuity theory & $\begin{array}{l}\text { Theory asserts that the different habitats in the human respiratory tract from the nasal and oral cavities to upper } \\
\text { and lower airways are contiguous and bacteria in the lower airway would be indistinguishable from the upper airway } \\
\text { bacteria }\end{array}$ \\
\hline $\begin{array}{l}\text { Whole genome sequencing } \\
\text { (metagenomic sequencing) }\end{array}$ & $\begin{array}{l}\text { Sequencing all the DNA in biological sample which can detect all genes constituting the collective metabolic } \\
\text { and functional capacity of the targeted habitat }\end{array}$ \\
\hline
\end{tabular}

DNA: Deoxyribonucleic acid; rRNA: Ribosomal ribonucleic acid.

To address the concern of upper airway contamination of lower airway sampling, Charlson et al. ${ }^{21}$ investigated the microbiome in both the upper and lower respiratory tract in six healthy individuals employing double bronchoscope sampling (Fig. 1), a technique developed by our group for conventional microbiology studies of the lower airway microbiome. In this technique, the upper and lower airways are anesthetized with one scope, and subsequently a second 

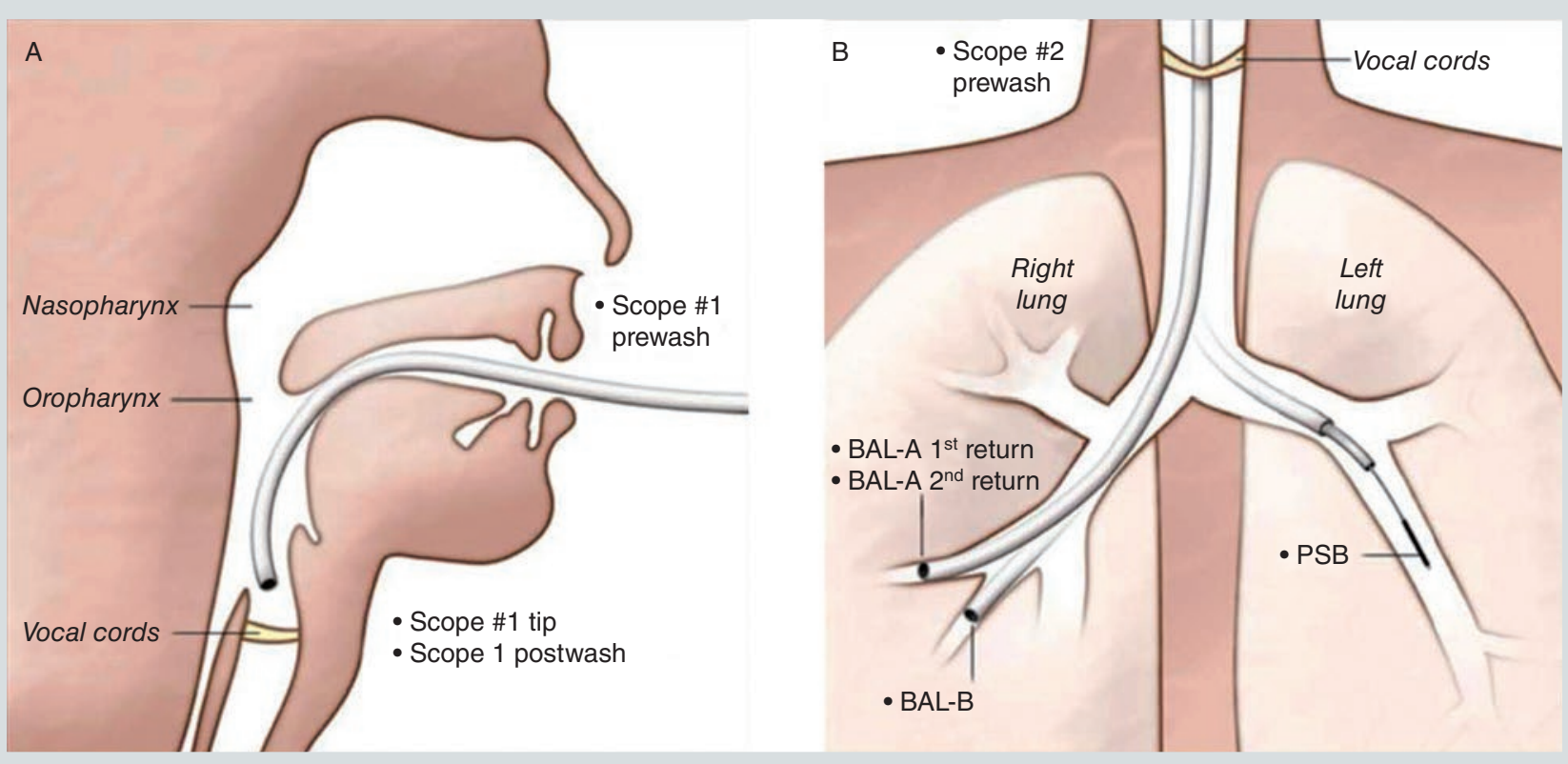

Figure 1. Double bronchoscope method. Localized anaesthesia and secretion management with bronchoscope \#1. A: After oropharyngeal anaesthesia administration, bronchoscope \#1 introduced transorally and guided to the glottis; local anaesthesia administered and visible secretions aspirated. The bronchoscope \#1 then removed from airway. B: Airways sampling via the bronchoscope \#2. Bronchoscope \#2 was introduced transorally, and biological samples then obtained from lower airways (reproduced with permission from Charlson ES et al. ${ }^{21}$ ).

BAL: bronchoalveolar lavage; PSB: protected specimen brush.

scope with an unused and therefore uncontaminated channel is used to obtain bronchial brushings and lavage ${ }^{23}$. Inclusion of control measurements of reagents and saline washes of bronchoscopes prior to sampling characterized the contribution of environmental contamination to the observed lower airways microbiome. Congruent with published studies, the lower airway samples had microbiota; however, the bacterial counts were much lower, in the range of 100-10,000. Low levels of bacterial DNA were seen in control samples, but these were in the range of 10-100 bacteria/ml. Comparison of the oropharyngeal swab and the bronchial brushings microbiome discovered a distinctly less abundant microbiome in the lower respiratory tract compared to the upper respiratory tract. There was more diversity and representation of bacterial lineages in the lower airways versus the upper respiratory tract. Although several unique OTUs were found only in the lower respiratory tract versus the upper respiratory tract, none were shared amongst different subjects.

Subsequent studies have supported the observations of Charlson et al. ${ }^{21}$, and now there is consensus that there is a healthy lung microbiome, albeit relatively sparse in comparison to that of the upper airway. It is likely that the healthy human lung microbiome represents transitory presence of micro-aspirated upper airway microbial flora that is in the process of being cleared by normal lung defence mechanisms ${ }^{24}$. The acronym TBNR, which represents 'transient but not resident' appears to be a fairly accurate characterization of the healthy lung microbiome ${ }^{25}$. 


\section{IN COPD, DYSBIOSIS MAY LEAD TO MALADAPTIVE INFLAMMATORY RESPONSES AND SUBSEQUENTLY TO FURTHER CHANGES IN THE LUNG MICROBIOME}

In contrast to the "sterile" healthy lung, COPD airways often contain bacteria, including potential respiratory pathogens based on previous traditional culture based studies of the lower respiratory tract in stable COPD ${ }^{23,26}$. Furthermore, this bacterial milieu is associated with inflammatory responses, radiological changes of bronchiectasis, pathological changes of a local immune response and increased daily COPD related symptoms ${ }^{27}$. These observations support the vicious circle hypothesis (Fig. 2) ${ }^{28}$. The vicious circle hypothesis proposes that alterations in innate lung defence induced by inhalational exposure due to tobacco smoke or biomass smoke allow particular pathogenic bacteria that normally enter the lower respiratory tract with other commensal organisms by micro-aspiration, to persevere and flourish. These bacteria, signalling through various pathogen recognition receptors on airway epithelial and immune cells, induce inflammation, which represents an attempt by the lung to clear the infection. However, the inflammation induces further damage to innate lung defence mechanisms, allowing further bacterial persistence and proliferation and thus establishing the vicious circle. This circle of chronic infection and inflammation contributes to the progressive lung damage and loss of function that is characteristic of COPD, often even after the primary insult (e.g. tobacco smoke) is removed $^{29}$. Improvements in molecular characterization of bacteria and studies of host immunological responses to these pathogens led to the establishment of another important role of bacteria in COPD, as a cause of about $50 \%$ of exacerbations ${ }^{28}$.

The vicious circle hypothesis as described above has focused on well-described potential respiratory pathogens as the major drivers of disease. Given our increasing knowledge of lung microbiomes in healthy and diseased lungs the vicious circle hypothesis has been revised (Fig. 2). Insults such as tobacco smoke that impair innate immune defences lead to changes in the abundance, taxonomic composition and/or phylogenetic diversity of the lung microbiome. This microbiome imbalance or dysbiosis leads to maladaptive inflammatory responses, further impairment of lung defences and further derangements of the lung microbiome, setting up the vicious circle with its attendant consequences. Substantial acute changes in the airway microbiome, for example by introduction of a new strain of a respiratory pathogen, lead to larger inflammatory responses, which present clinically as exacerbations of COPD. It is likely the contribution of the vicious circle to COPD progression and pathogenesis varies among individuals, with a larger contribution in those individuals with a greater deviance from normal in their innate immune defences and/ or the inflammatory response to the microbiome.

Supportive evidence for the vicious circle hypothesis and bacterial exacerbation mechanisms based on conventional culture techniques has been reviewed in earlier publications ${ }^{27,28}$. Below we will discuss studies using microbiome techniques examining this hypothesis $^{30}$. 


\section{Vicious circle hypothesis revised}

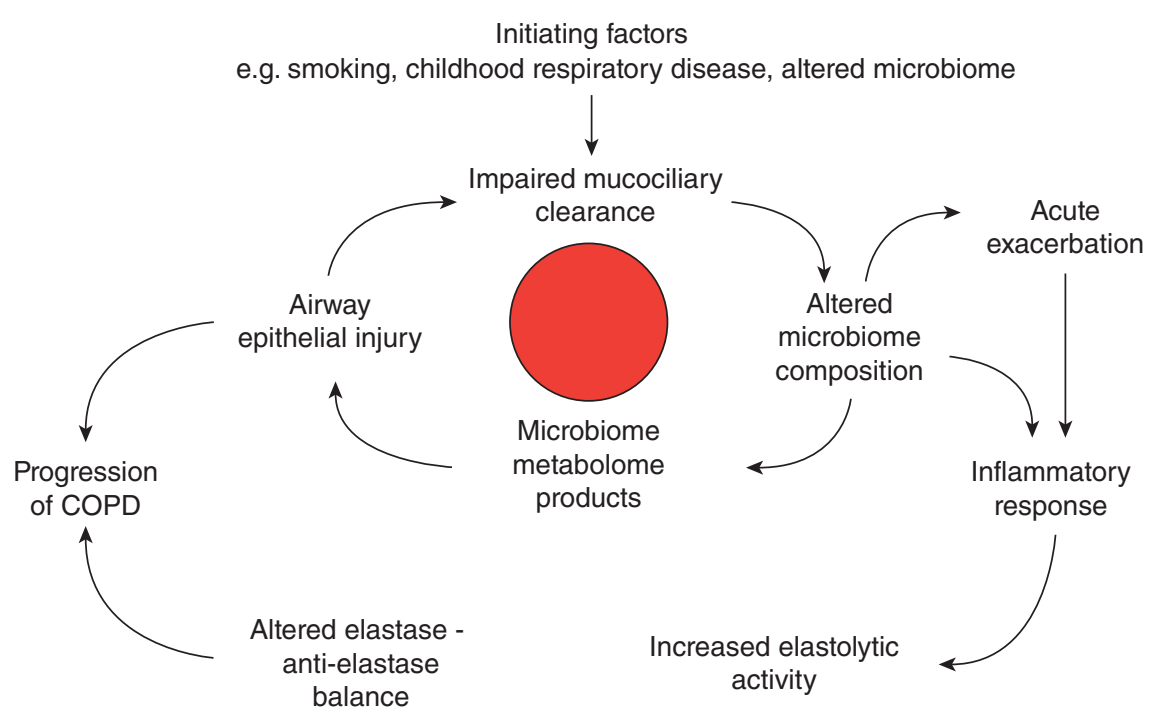

Figure 2. The vicious cycle in chronic obstructive pulmonary disease (COPD) pathogenesis. After the initial insult impairs innate lung defence, changes in the microbiome perpetuates a cyclical sequence of events that contributes to the persistent inflammation and microbiome changes that are characteristic of COPD (reproduced with permission from Sethi S et al.28).

\section{CHANGES IN COPD MICROBIOME BECAUSE OF THE ALTERED LUNG MILIEU}

One prevalent theme in the evaluation of the human microbiome is that different human anatomic locations constitute different "islands" of habitation and differ in time and location, as described in the island biogeography theory $^{7}$. However, a countervailing theory based on topological continuity of the airways asserts that the different habitats in the human respiratory tract from the nasal and oral cavities to upper and lower airways are contiguous and bacteria in the lower airway would be indistinguishable from the upper airway bacteria $^{31}$.
Studies in healthy controls support the topological continuity theory as shown by Charlson et al. ${ }^{21}$ and recently by Bassis et al. ${ }^{32}$ In the latter study, the microbiome from the oral cavity, nasal cavity, both lungs and the stomach from 28 healthy subjects were compared to determine the contribution to the microbiome of the lower aerodigestive sites from the upper sites ${ }^{32-34}$. They found that the nasal cavity microbiome is distinct, however, the mouth was a major source of the microbiome community members of the gastric and BAL microbiome.

In contrast to the healthy lung microbiome which is similar to the oral microbiome, studies describe disparate upper and lower airway 
microbiomes in COPD which supports the substantial influence of the lung micro-environment on the microbiome in COPD. Though the microbiome is likely initially derived from the upper airway in COPD, there must be substantial changes in its composition from interactions with the disease induced local micro-environment. Furthermore, this local micro-environment likely differs from the lung heterogeneity characteristic of COPD, resulting in corresponding variation in the microbiome. Such variability has been described by Cabrera-Rubio et al. ${ }^{35}$, when they compared sputum, bronchial aspirate, BAL and bronchial mucosa biopsy microbiomes in six subjects with moderate COPD. The bronchial aspirate and sputum microbiomes were distinct from the lavage and bronchial mucosa biopsy microbiomes, which were similar to each other. In the approximately 4-8 airway sites per lobe sampled within six explanted lungs from patients with severe COPD, Erb-Downward et al. ${ }^{22}$ found significant heterogeneity driven by variable dominance of Pseudomonas, Haemophilus, or Stenotrophomonas genera.

The lung tissue microbiome from multiple sites of surgically resected lungs from non-smokers, smokers, very severe COPD and cystic fibrosis (CF) ( $n=8$ in each group) was studied by Sze et al. ${ }^{36}$, and they found a sparse microbiome in all the lung tissue samples. The COPD samples did not differ in bacterial abundance from the non-smoker and smoker control samples, but did differ in composition.

\section{COPD SEVERITY IS INVERSELY RELATED TO MICROBIOME DIVERSITY}

Reduced diversity with increasing severity of COPD was observed by Garcia-Nuñez et al. ${ }^{39}$, in their investigation of the sputum microbiome in moderate to severe versus very severe COPD. Galiana et al. ${ }^{37}$ found diminished sputum microbiome alpha diversity in severe COPD ( $\mathrm{n}=10)$ compared to moderate to severe disease $(n=9)$, with overrepresentation of the Actinomyces genus in the latter group. In order to determine whether colonization with Pseudomonas aeruginosa altered the sputum microbiome of COPD, during stable disease as well as during exacerbations, Millares et al. ${ }^{38}$ studied sixteen individuals with severe or very severe COPD. The subjects were grouped into those with $(n=5)$ and those without ( $\mathrm{n}=11$ ) Pseudomonas aeruginosa colonization. Interestingly, biodiversity and other community characteristics of the microbiome were not different in the two groups during stable disease and exacerbation.

The observation in COPD of decreasing microbiome diversity associated with increasing disease severity parallels observations in other chronic airway diseases such as bronchiectasis and CF. While introduction of Pseudomonas aeruginosa does not cause alteration of microbiome diversity in COPD, it has been associated with adverse long-term outcomes in COPD. It is yet to be determined whether reduced diversity is related to other clinical COPD attributes such as increased inflammation, frequent exacerbations and disease progression $^{39}$.

\section{AIRWAYS MICROBIOME CHANGES WITH ONSET AND TREATMENT OF COPD EXACERBATION}

Acute COPD exacerbation (AECOPD) pathogenesis was characterized by conventional 
culture studies, in combination with immunological and inflammatory profiling, in human samples and animal models for the prior two decades ${ }^{40}$. Yet, in approximately half the AECOPD with the clinical characteristics of bacterial involvement, bacterial pathogens are not recovered by traditional culture methods. A limitation of conventional culture techniques is that identification of known pathogens is the main objective, rather than discovery of new pathogens. Next generation sequencing aided analysis of AECOPD are yielding novel findings. We analysed the sputum microbiome in serial monthly sputum samples collected in a longitudinal study of bacterial infection in COPD through 12 AECOPD episodes ${ }^{41}$. In the study, two sputum samples were collected prior to the exacerbation, one collected when the patient presented with the exacerbation but before treatment, and two samples collected after the exacerbation were clinically resolved ${ }^{23,28,42}$. Microbial DNA from sputum samples were hybridized to a DNA microarray ${ }^{43}$. The two samples from pre-exacerbation stable disease had similar microbiomes within the same patient, suggesting short-term stability of the microbiome in stable COPD. During AECOPD, no changes in community richness, evenness and diversity of the sampled microbiome were observed. Nevertheless, substantial taxonomic composition variations were associated with an increase in Proteobacteria and a decrease in Actinobacteria, Clostridia, and Bacteroidia. Also, when known pathogens such as Haemophilus influenzae increased during AECOPD, closely related bacterial taxa in the phylogenetic tree were also enriched, while those taxa that were phylogenetically distant declined. These findings suggest enrichment of taxa closely related to a dominant pathogen could contribute to AECOPD pathogenesis.

Systematically collected post-exacerbation samples provided an opportunity to assess treatment effects on the COPD airway microbiome. There was increased bacterial abundance in these post-exacerbation samples with only oral steroid treatment of the exacerbation, with enrichment of many taxa including members of Proteobacteria, Bacteroidetes, and Firmicutes. In contrast, individuals treated only with antibiotics reduced bacterial abundance, specifically Proteobacteria members. After both steroids and antibiotics were employed during an AECOPD treatment regimen, a variegated effect on the airway microbiome was seen (Fig. 3).

Similar findings of microbiome variations with exacerbation was observed with an experimental human model of rhinovirus-induced AECOPD, by Molyneaux et al. ${ }^{44}$ where rhinovirus was inoculated intranasally in 31 human subjects (control subjects $=17$, Moderate COPD subjects $=14$ ). The sputum was collected prior (Day 0) and 5, 15, and 42 days after rhinovirus inoculation, in which rhinovirus levels, the microbiome, bacterial abundance and inflammatory parameters were assessed. In the COPD group, bacterial abundance was significantly increased from baseline, but not in the control group, and this increase correlated with elevated neutrophil concentration and neutrophil elastase levels. As opposed to no significant microbiome changes in the control group, the COPD group had significant microbiome composition dysbiosis, with a decline in Firmicutes and Bacteroidetes, and an increase in Proteobacteria, including a $21 \%$ increase in 
A

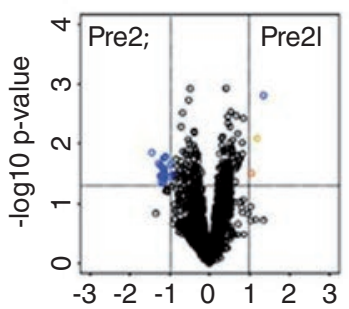

Pre2 vs. Esac

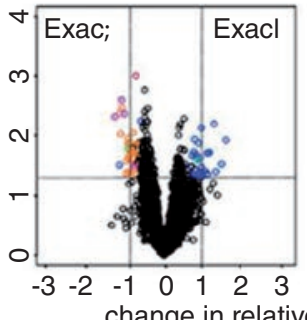

change in relative abundance $(\log 2)$
Exac vs. Post 1

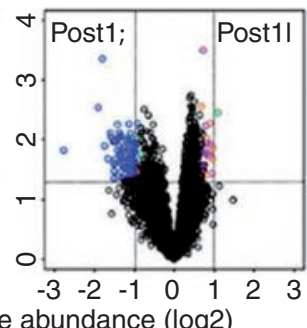

Post1vs. Post2

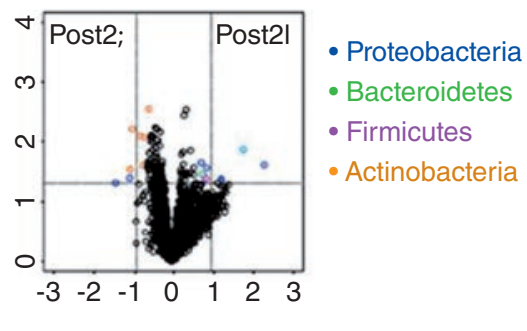

B

Exac vs. Post 1

post1 vs Post2

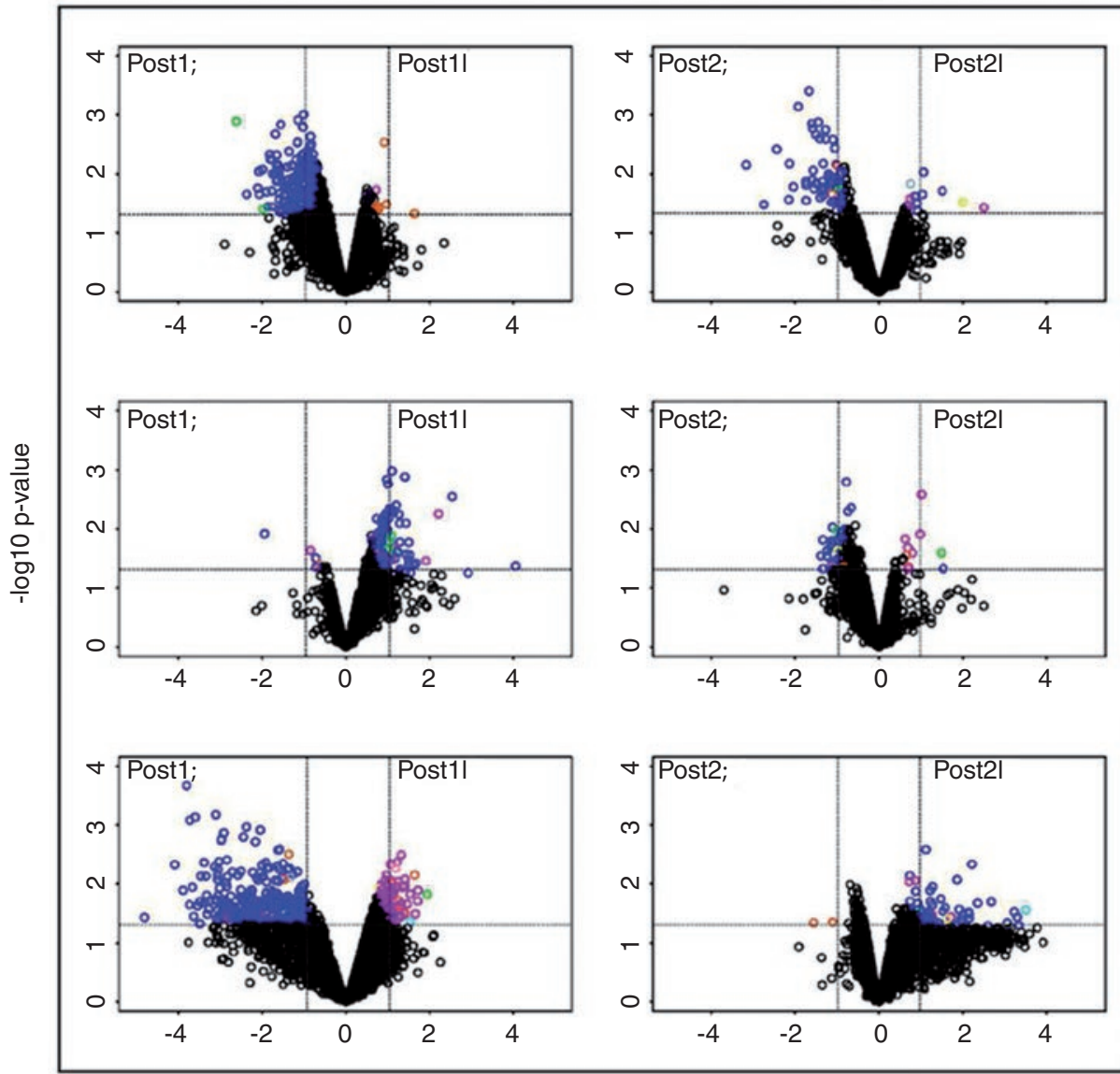
Antibiotics only

Steroids

only

Both antibiotics \& steroids

Figure 3. Airway microbiome dynamics after treatment. A: Volcano plots indicating taxa that are significantly increased (upper right quadrant) or decreased (upper left quadrant) in the pairwise comparisons indicated, using moderated t-test models (R package limma). Results shown are from analyses of all subjects. Dashed lines, significant false discovery rate adjusted $P$ values and changes in relative abundance of at least 2 -fold, or log2 equal to 1. Taxa exhibiting significant changes are coloured by phylumlevel classification, as shown. Note that in addition to the highlighted taxa, many other microbiota members exhibited smaller-scale changes in abundance, which cumulatively may contribute importantly to microbiome community function. B: Changes in the relative abundance of taxa from exacerbation (exac) to Post1 and Post1 to Post2, segregated by type of exacerbation treatment (antibiotics only, systemic corticosteroids only, or both; $\mathrm{n} 4$ subjects in each treatment group) (reproduced with permission from Huang YJ et al. ${ }^{41}$ ). 
Haemophilus and a 9.5\% increase in Neisseriaceae.

\section{LUNG MICROBIOME DIVERSITY AND RICHNESS DECLINE WAS ASSOCIATED WITH GREATER EMPHYSEMA AND GREATER IMMUNE CELL INFILTRATION}

The vicious circle hypothesis suggests that the host immune response to the microbiome dysbiosis is maladaptive and contributes to progression of COPD. Evidence supporting the process utilizing new sequencing techniques studying the airways microbiome was published recently. In an innovative study, Sze et al. ${ }^{45}$ examined the microbiome of five lungs from subjects with GOLD grade 4 (very severe airflow obstruction) disease, and four healthy donor lungs not used for transplantation. Alveolar destruction was quantified by measuring alveolar surface area of the tissue samples imaged by computed tomography (CT) scan and correlated to immunohistochemistry of histological lung tissue sections quantifying the immune cells. The relationship between microbiota, inflammatory immune cell infiltration and alveolar destruction was analysed by correlating the microbiome, immunohistological and CT scan data. Declining microbiome diversity and richness was associated with increased alveolar destruction and greater CD4 cell infiltration. Expansion of Proteobacteria and Actinobacteria phyla distinguishes the COPD group from the control group, while Bacteroidetes and Firmicutes had a greater representation in control lungs versus the COPD lungs. These observations suggest that changes in the microbiome, specifically increases in Proteobacteria and Actinobacter phyla drive the progression of COPD.

\section{CYSTIC FIBROSIS}

$\mathrm{CF}$ is regarded as the prototypical lung disease that is driven by chronic bacterial infection. This has prompted multiple microbiome studies to understand the role the airways microbiome in CF airways.

\section{DYSBIOSIS IN THE AIRWAY MICROBIOME IS PREVALENT IN CF AND CHANGES WITH AGE}

Sibley et al. ${ }^{46}$. initial study examining the microbiome in CF airways revealed a complex airway microbiome with 16s rRNA gene sequencing, and $46 \%$ of the bacterial groups identified in the samples were comprised of obligate anaerobes ${ }^{46}$. A microbiome analysis of the different areas of lung tissue obtained from a lobectomy performed on a 3-year-old CF patient revealed a spatial variation in the composition of the microbiota ${ }^{47}$. A $16 \mathrm{~S}$ rRNA PCR amplification coupled with a rRNA microarray, the G3 Phylochip Array, was used to analyse BAL and oropharyngeal swabs from 13 children with $\mathrm{CF}$ and 9 children without $\mathrm{CF}^{48}$. The $\mathrm{CF}$ cohort had increased diversity and increased abundance of Prevotella veroralis, CW040 and a Corynebacterium compared to the non-CF cohort, indicating a disruption of the CF respiratory microbiome early in life.

In a relatively large study, Coburn et al. examined the sputum microbiome by $16 \mathrm{~S}$ rRNA gene sequencing in $269 \mathrm{CF}$ patients ranging from age 4 to 64 . The found greater microbial 
diversity in the younger age group ( $<18$ years) and a distinct dominance (most abundant genus) of Streptococcus, Haemophilus, Pseudomonas, Staphylococcus and Achromobacter genera in the pediatric population as opposed to the adult population which had Pseudomonas, Burkholderia, Streptococcus, Haemophilus and Staphylococcus as the dominant genera ${ }^{49}$.

An Italian study by Bacci et al..$^{50}$ compared the sputum microbiome in $\mathrm{CF}$ patients with a substantial decline in lung function to those in whom the lung function remained stable. Decreased microbial diversity was seen in the group with substantial decline in lung function, while an increase in Staphylococcus was seen in the stable group.

In summary, the lung microbiome is disrupted in CF early in life, and changes in structure and composition with age and progression of disease.

\section{AIRWAYS MICROBIOME IN CF EXACERBATIONS}

Though several studies have examined changes in the airway microbiome with CF exacerbations, the findings have been inconsistent. When Fodor et al. ${ }^{51}$ examined the microbiome of 23 adult CF patients in expectorated sputum and oral wash samples obtained during acute exacerbation, after antibiotic treatment, and during stable disease, they found that $60 \%$ of the sequences were from Pseudomonas and Burkholderia. However, the microbial composition was similar in the three sample collection time periods, indicating that exacerbations in $\mathrm{CF}$ were not related to acute changes in the airways microbiome.
On the other hand, when Carmody et al. ${ }^{52}$ explored the microbiome of 68 paired sputum samples at baseline and during exacerbation from $28 \mathrm{CF}$ patients, the abundance of the $\mathrm{Ge}$ mella bacterial genus was increased during exacerbation compared to baseline samples. Reid et al. ${ }^{53}$ microbiome analysis of sputum from $\mathrm{CF}$ patients with pulmonary exacerbation compared to CF patients without exacerbation revealed an alteration of the anaerobic bacterial population, particularly an increase of the Chrysiogenales genus, which was also associated with elevated levels of anaerobic metabolites: pyruvate, lactate and putrescine.

A subsequent metagenomics analysis of $\mathrm{CF}$ sputum samples collected longitudinally amongst three patients before, after and during exacerbation reveals unique diverse microbiota composition that changes over time ${ }^{54}$. The microbiota corresponding to the different individuals were associated with variable microbial metabolic potentials and dynamic antibiotic resistance profiles.

\section{CHANGES IN CF MICROBIOME WITH THERAPY}

On $16 \mathrm{~S}$ gene sequencing of $\mathrm{CF}$ sputum samples collected during a pulmonary exacerbation at three time points (before intravenous antibiotic administration, at day 3 of intravenous antibiotic administration, and day 8-10 day of intravenous antibiotics) from 23 individuals, bacterial composition was the same before and after treatment. However, there was a decrease in Pseudomonas abundance with a transient increase in microbial diversity on day 3 , that dissipates by the second week of intravenous antibiotics ${ }^{55}$. 
Oral supplementation with Ferrous sulphate in a randomized, double-blind, trial in CF patients did not change the diversity of the microbiome nor the abundance of pathogenic genera, Pseudomonas, Staphylococcus, Haemophilus, Rothia and Achromobacter ${ }^{56}$. This finding allays the concern that supplemental iron could cause pulmonary exacerbations or increase Pseudomonas abundance ${ }^{57}$.

A therapy targeted against the CF transmembrane conductance regulator (CFTR) G551D mutation, Ivacaftor, has improved FEV1 in selected CF populations, and was shown to alter the sputum microbiome analysed with a statistically significant increase in Porphyromonas and significant decrease of Streptococcus bacteria $^{58}$.

\section{NEW APPROACHES IN CF AIRWAYS MICROBIOME CHARACTERIZATION}

The first metagenomics analysis of CF sputum samples from two individuals aged 14 and 17, revealed high abundances of Haemophilus, Staphylococcus and Streptococcus species, with relatively few fungal species identified ${ }^{59}$. A metagenomics and metatranscriptomic (RNA sequencing) analysis of CF sputum samples conducted by Quinn et al. ${ }^{60}$, revealed the microbiota had significant enrichment of amino acid metabolism, folate biosynthesis and lipoic acid biosynthesis. Further analysis of the metabolism of the microbiota may allow for novel therapeutics targeted against essential metabolic pathways of the CF microbiome.

A study by Kim et al ${ }^{61}$. utilized high-throughput sequencing of the ribosomal RNA internal transcribed spacer 1 (ITS1), a common method to sequence fungal communities, and found that Candida and Aspergillus species were the dominant fungi in CF sputum samples.

In summary, lung microbiome changes can be seen early in life in CF, with further changes associated with age, disease progression and treatment. The impact of these changes in the microbiome on the manifestations and course of this disease are still to be understood.

\section{NON-CF BRONCHIECTASIS (NCFB)}

Though there is longstanding recognition of the role of the lung microbial flora in driving structural changes in the lung, such as bronchiectasis, as described in the vicious circle hypothesis, there has only been limited exploration of the microbiome in bronchiectasis.

\section{AIRWAY MICROBIOME IN NCFB}

Conventional microbiology studies, such as the one by McDonnell et al. ${ }^{62}$, had revealed a diverse microbial flora in sputum samples from patients with $\mathrm{NCFB}^{62}$. The specific focus of these studies was usually aerobic flora and conventional respiratory pathogens. Though Haemophilus influenzae was often the most common pathogen isolated, the isolation of Pseudomonas aeruginosa was associated with worse lung function and clinical outcomes. When Rogers et al. ${ }^{63}$ applied $16 \mathrm{~s}$ microbiome sequencing methods to characterize sputum samples from NCFB patients, they found that along with H. influenzae and P. aeruginosa, Veillonella, Prevotella, Streptococcus and Neisseria were also often the dominant taxa ${ }^{63}$. Increased microbiota diversity was associated with better lung 
function and less airway and systemic inflammation, while these measures were worse when any taxa was dominant.

\section{EFFECT OF TREATMENT AND EXACERBATIONS ON THE AIRWAY MICROBIOME IN NCFB}

Macrolide antibiotics are often used to prevent exacerbations of NCFB. The effect of such treatment on the sputum microbiome was examined by Rogers et al. ${ }^{64,65}$ in the BLESS trial with Bronchiectasis and low dose erythromycin (BLESS trial: ACTRN12608000460303) trial, where they had access to 44 samples before and after erythromycin treatment for 12 months, and 43 control samples where patients received placebo. A worrisome emergence of $P$. aeruginosa was seen only in the erythromycin group if their baseline samples did not contain this pathogen.

In a striking similarity to descriptions in COPD and CF, there were no major changes in the airway microbiome community structure with exacerbation ${ }^{66}$, though this study was limited in power. Changes in composition of the microbiome at exacerbation were seen, and larger studies are needed to better characterize the impact of exacerbations and their treatment on the microbiome in NCFB.

\section{ASTHMA}

The complexity of asthma phenotypes and endotypes is now well recognized, and several investigations have examined for associated changes in the lung microbiome. Insights in to the role of upper airway and gut microbiomes early in life in determining the emergence of asthma have grown and are discussed below.

\section{AIRWAYS MICROBIOME DIFFERS IN ASTHMA AND HAS FUNCTIONAL CORRELATIONS}

The first study in asthma by Hilty et al. ${ }^{20}$ study using 16S rRNA based PCR amplicon analysis revealed that asthmatic airways had reduced Bacteriodetes (particularly Prevotella) and increased Proteobacteria (particularly Haemophilus) compared to controls. However, this study had methodological limitations as discussed above.

Huang et al. examined adult asthmatics and healthy controls who underwent bronchial epithelial brushings during a study examining macrolide therapy to reduce airway bacteria to improve asthma control. Asthmatic subjects had greater microbial diversity than controls, and bronchial hyperresponsiveness was inversely correlated to bacterial diversity in the asthmatic cohort ${ }^{67}$. The relative abundance of particular bacterial families of the Proteobacteria phylum, members of the Comamonadaceae, Sphingomonadaceae, Oxalobacteraceae, correlated with the degree of bronchial hyperresponsiveness. Marri et al. ${ }^{68}$, showed different microbiome composition between asthmatic and non-asthmatic adults utilizing 16S rRNA gene sequencing of DNA extracted from induced sputum. Increased Proteobacteria and lower Firmicutes and Actinobacteria proportions in the asthmatic airways compared to the health controls were seen.

A metatranscriptomic or shotgun RNA sequencing analysis of nasal samples from 
asthmatic and non-asthmatic participants of the Asthma Severity Modifying Polymorphisms (AsthMaP) project suggests that asthma nasal microbiome is less diverse than healthy controls $^{69}$. In this study there were more Proteobacteria and less Firmicutes in the asthmatic nasal microbiome, and a dominance of the Proteobacterium, Moraxella catarrhalis in the asthmatic samples. A secondary analysis of the microbe-host interactions found an increased expression of microbiome adhesion genes. Furthermore, the presence of Proteobacteria was positively associated with an upregulation of a host pro-inflammatory cytokine, IL-1 $\alpha$, suggesting compositional changes in the microbiome can lead to functional changes that modulate the host inflammatory response $^{70}$.

Alterations in the microbiome with the severity and control of asthma have now been described. In a recent study by Huang et al. ${ }^{71}$ bronchial brushings revealed dysbiosis of the airways microbiome in severe and mild-moderate asthmatics, when compared to healthy controls. Proteobacteria were associated with worse asthma control, while Actinobacteria was an indicator of steroid responsiveness. Zhang et al. ${ }^{72}$ examined the induced sputum microbiome in 26 severe asthmatic, 18 mild-moderate asthmatic, and 12 healthy adults and found that Proteobacteria was more common in mild-moderate asthma group while Bacteroidetes and $F u$ sobacteria were reduced in the mild-moderate and severe asthma groups.

In summary, the airway microbiome is indeed altered in asthma and could have a role in determining airway hyper-responsiveness, airway inflammation, response to medications and the level of asthma control.

\section{MICROBIOME EXPOSURE IN EARLY CHILDHOOD CONTRIBUTING TO ASTHMA PREDISPOSITION}

The premise that sterile environments may predispose patients to asthma, while early childhood exposures to diverse microbial milieu prevents asthma acquisition, is the basis of the hygiene hypothesis ${ }^{73}$. The finding that greater microbial diversity is inversely related to airway hyper-responsiveness is congruent with the recent revision of the hygiene hypothesis that early life exposure to diverse microbes diminishes the risk of developing asthma ${ }^{74}$. A complementary theory, the infectious asthma hypothesis asserts decreased microbiome and exposure to specific pathogenic microbes early in life cause lung injury predisposing individuals to repeated airway damage and ultimately activates a strong Th2 immune response to environmental antigens ${ }^{74}$ (Fig. 4).

Evidence to support this revised hygiene hypothesis includes observations of the low incidence of asthma in Amish populations in the US, as in this farming community, childhood contact with farm animals exposes them diverse microbiota ${ }^{73}$. The Prevention of Allergy-Risk Factors for Sensitization in Children Related to Farming and Anthroposophic Lifestyle (PARSIFAL) and Multidisciplinary Study to Identify the Genetic and Environmental Causes of Asthma in the European Community (GABRIELA) study groups describe decreased rates of asthma in children growing up in farms in central Europe, compared to children located in the same region not living on farms. Furthermore, they found an inverse correlation between the probability of developing asthma and the number of bacteria and fungi groups detected via $16 \mathrm{~S}$ rRNA and traditional 
Early-life airway microbial exposure and development of asthma

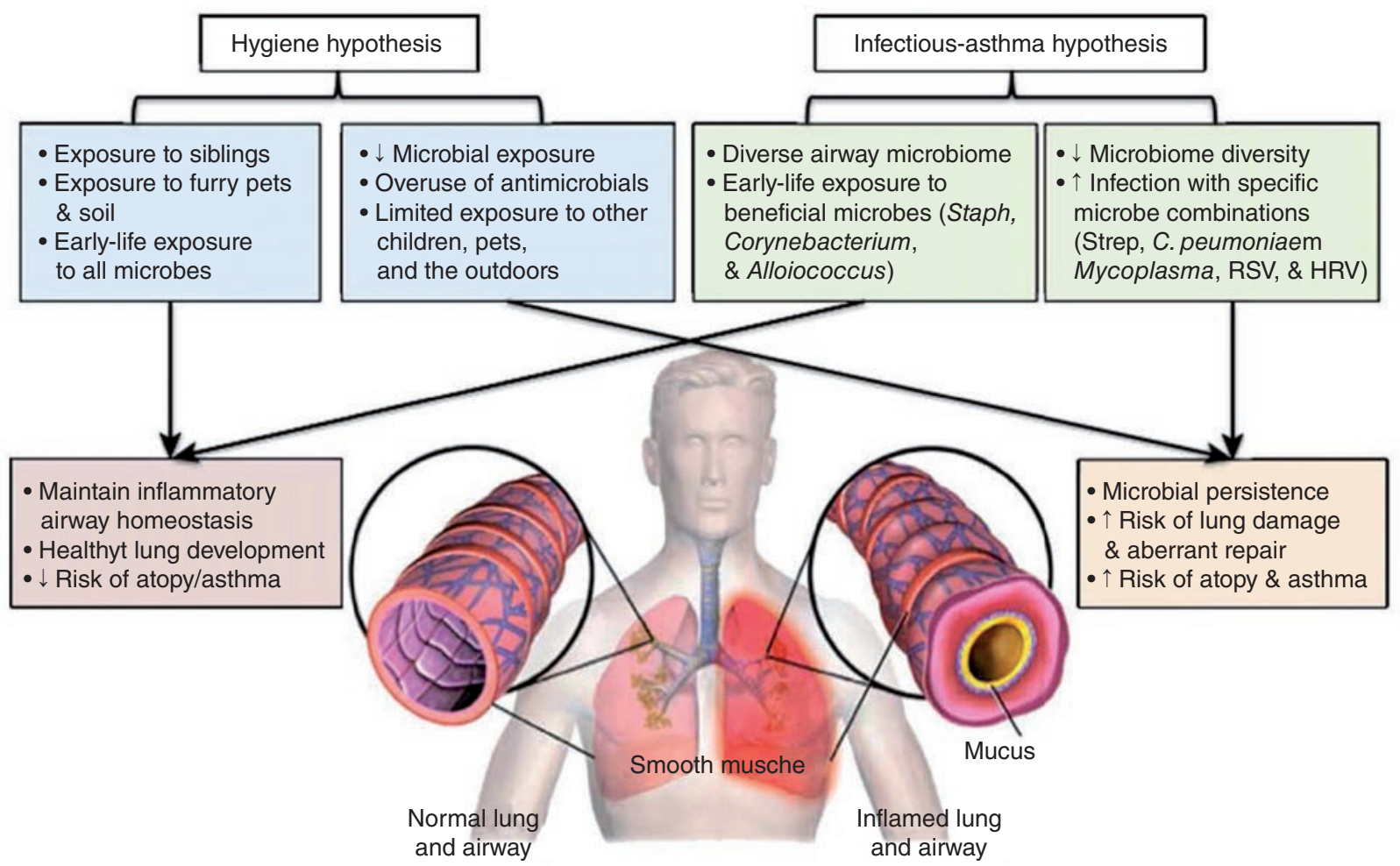

FiguRE 4. Hygiene and infectious asthma hypothesis early life airway microbial exposure and development of asthma (reprinted from Trends in Microbiology, Webley et al. ${ }^{74}$, Copyright (2015), with permission from Elsevier).

culture techniques in dust collected from the respective household ${ }^{75}$. In order to further understand the effect of the impact of the environmental microbial contact on the development of asthma, Stein et al. ${ }^{76}$ studied two distinct, endogamous farming subgroups in the US, the Amish and Hutterites, two populations that are genetically more similar than other European populations. They have very similar lifestyles and risk factors for asthma, except the Amish use traditional farming methods, with frequent contact with farm animals, while the Hutterites live on highly mechanized communal farms. The Amish have a quarter of the incidence of asthma compared to the Hutterites (5 versus 21\%). Dust collected via electrostatic dust collectors and mattresses from the Amish homes had endotoxin levels nearly seven times higher than the Hutterite homes. The microbial composition between the two groups was different with increased relative abundance of Bartonellaceae in the Amish sample compare to the Hutterite sample. When extracts of dust collected from 
the floors of the living rooms of the ten homes from each community was administered intranasally to a murine ovalbumin model of allergic asthma, only the Amish dust prevented IgE elevation, airway eosinophilia and airway hyperresponsiveness.

\section{INTERSTITIAL LUNG DISEASE}

As with other chronic lung diseases, the airways microbiome in interstitial lung disease (ILD) has also been studied. However, one major confounder with the ILD airways is the heterogeneous prevalence of traction bronchiectasis, which could lead to changes in airway microbiome composition that are more a result of the fibrosis rather than a cause.

\section{AIRWAYS MICROBIOME IS DIFFERENT IN ILD VERSUS HEALTH SUBJECTS}

The COMET-IPF (Correlating Outcomes with biochemical Markers to Estimate Time-progression in Idiopathic Pulmonary Fibrosis) investigators used principal component analysis of $16 \mathrm{~S}$ rRNA gene sequencing of DNA extracted from BAL from individuals with idiopathic pulmonary fibrosis (IPF) to identify two OTU clusters associated with disease progression: Streptococcus and Staphylococcus $^{77}$. A subsequently published, prospective study examined the BAL microbiome in 65 subjects with IPF compared to 44 healthy controls and 17 subjects with moderate COPD ${ }^{78}$. The BAL from IPF subjects had $>2$ times the copies of $16 \mathrm{~S}$ rRNA gene/ml, signifying larger bacterial loads than both control and COPD groups. However, there were no controls included to account for oral contamination. The bacterial burden in IPF was associated with a known host factor associated with developing IPF, the rs35705950 polymorphism in the promoter of the mucin gene MUC5B genotype, which has been implicated in bacterial clearance ${ }^{79,80}$. Although diversity was not significantly different between the three groups, Haemophilus, Streptococcus, Neisseria, and Veillonella genera were more abundant in the IPF microbiome versus the control group. In contrast to these findings, Garzoni et al. ${ }^{81}$ characterized the airway microbiome in BAL and oropharyngeal swabs in ILD and found no dysbiosis amongst their cohorts.

\section{CONCLUSION}

A sparse transient microbiome in healthy individuals that exhibits significant dysbiosis with the development of chronic lung disease is now firmly established. Further changes in the microbiome appear with a change in state from stable to exacerbation in chronic lung diseases. Imbalance of the airway microbiome may lead to maladaptive host responses that predispose individuals to chronic lung disease. We are only beginning to understand the functional implications of this dysbiosis and its contribution to disease progression, precipitation of exacerbations and impact on therapeutic interventions in chronic lung diseases ${ }^{82}$. Increasing application of new techniques such as whole genome sequencing and metatranscriptomics with larger cohorts sampled over longer time periods should provide us greater insights. Methodological advances in obtaining microbiome data have outpaced the development of robust and innovative tools to interpret the microbiome data. 


\section{CONFLICT OF INTEREST}

\author{
Dr. Sanjay Sethi and Dr. Manoj J. Mammen \\ have nothing to disclose.
}

\section{REFERENCES}

1. Group NHW, Peterson J, Garges S, et al. The NIH Human Microbiome Project. Genome research. 2009;19:2317-23.

2. Sender R, Fuchs S, Milo R. Revised Estimates for the Number of Human and Bacteria Cells in the Body. PLoS Biology. 2016;14:e1002533.

3. Qin J, Li R, Raes J, et al. A human gut microbial gene catalogue established by metagenomic sequencing. Nature. 2010;464:59-65.

4. Gill SR, Pop M, Deboy RT, et al. Metagenomic analysis of the human distal gut microbiome. Science. 2006;312:1355-9.

5. Dethlefsen L, McFall-Ngai M, Relman DA. An ecological and evolutionary perspective on human-microbe mutualism and disease. Nature. 2007;449:811-8.

6. Blaser MJ, Falkow S. What are the consequences of the disappearing human microbiota? Nature Rev Microbiol. 2009;7:887-94.

7. Turnbaugh PJ, Ley RE, Hamady M, et al. The human microbiome project. Nature. 2007;449:804-10.

8. Medini D, Serruto D, Parkhill J, et al. Microbiology in the post-genomic era. Nature Rev Microbiol. 2008;6:419-30.

9. D'Onofrio A, Crawford JM, Stewart EJ, et al. Siderophores from neighboring organisms promote the growth of uncultured bacteria. Chem Biol. 2010; 17:254-64.

10. Wade W. Unculturable bacteria--the uncharacterized organisms that cause oral infections. J R Soc Med. 2002;95:81-3.

11. Gilbert JA, Dupont CL. Microbial metagenomics: beyond the genome. Ann Rev Mar Sci. 2011;3:347-71.

12. Klindworth A, Pruesse E, Schweer T, et al. Evaluation of general 16S ribosomal RNA gene PCR primers for classical and next-generation sequencing-based diversity studies. Nucleic acids res. 2013;41:e1.

13. Chakravorty S, Helb D, Burday M, et al. A detailed analysis of $16 \mathrm{~S}$ ribosomal RNA gene segments for the diagnosis of pathogenic bacteria. J Microbiol Methods. 2007;69:330-9.

14. Aguirre E, Galiana A, Mira A, et al. Analysis of microbiota in stable patients with chronic obstructive pulmonary disease. APMIS. 2015;123:427-32.

15. Thomas T, Gilbert J, Meyer F. Metagenomics - a guide from sampling to data analysis. Microb Inform Exp. 2012;2:3.

16. Sharpton TJ. An introduction to the analysis of shotgun metagenomic data. Front Plant Sci. 2014;5:209.

17. Langille MG, Zaneveld J, Caporaso JG, et al. Predictive functional profiling of microbial communities using 16S rRNA marker gene sequences. Nat Biotechnol. 2013;31:814-21.

18. Morgan XC, Huttenhower C. Chapter 12: Human Microbiome Analysis. PLoS Comput Biol. 2012;8:e1002808.

19. Dickson RP, Erb-Downward JR, Martinez FJ, et al. The Microbiome and the Respiratory Tract. Annu Rev Physiol. 2016;78:481-504.

20. Hilty M, Burke C, Pedro H, et al. Disordered microbial communities in asthmatic airways. PloS one. 2010;5:e8578.

21. Charlson ES, Bittinger K, Haas AR, et al. Topographical continuity of bacterial populations in the healthy human respiratory tract. Am J Respir Crit Care Med. 2011;184:957-63.

22. Erb-Downward JR, Thompson DL, Han MK, et al. Analysis of the lung microbiome in the "healthy" smoker and in COPD. PLoS One. 2011;6:e16384.

23. Sethi S, Maloney J, Grove L, et al. Airway inflammation and bronchial bacterial colonization in chronic obstructive pulmonary disease. Am J Respir Crit Care Med. 2006;173:991-8.
24. Huffnagle GB, Dickson RP, Lukacs NW. The respiratory tract microbiome and lung inflammation: a two-way street. Mucosal Immunol. 2016.

25. Saeedi P, Salimian J, Ahmadi A, et al. The transient but not resident (TBNR) microbiome: a Yin Yang model for lung immune system. Inhal Toxicol 2015;27:451-61.

26. Soler N, Ewig S, Torres A, et al. Airway inflammation and bronchial microbial patterns in patients with stable chronic obstructive pulmonary disease. Eur Respir J. 1999;14:1015-22.

27. Rangelov K, Sethi S. Role of infections. Clin Chest Med. 2014;35:87-100.

28. Sethi S, Murphy TF. Infection in the Pathogenesis and Course of Chronic Obstructive Pulmonary Disease. N Engl J Med. 2008;359:2355-65.

29. Tager I, Speizer FE. Role of infection in chronic bronchitis. N Engl J Med. 1975;292:563-71.

30. Sethi S. Chronic obstructive pulmonary disease and infection. Disruption of the microbiome? Ann Am Thorac Soc. 2014;11 Suppl 1:S43-7.

31. Nguyen LD, Viscogliosi E, Delhaes L. The lung mycobiome: an emerging field of the human respiratory microbiome. Front Microb. 2015;6:89.

32. Bassis CM, Erb-Downward JR, Dickson RP, et al. Analysis of the upper respiratory tract microbiotas as the source of the lung and gastric microbiotas in healthy individuals. mBio. 2015;6:e00037.

33. Morris A, Beck JM, Schloss PD, et al. Comparison of the respiratory microbiome in healthy nonsmokers and smokers. Am J Respir Crit Care Med. 2013;187:1067-75.

34. Segal LN, Alekseyenko AV, Clemente JC, et al. Enrichment of lung microbiome with supraglottic taxa is associated with increased pulmonary inflammation. Microbiome. 2013;1:19.

35. Cabrera-Rubio R, Garcia-Nuñez M, Seto L, et al. Microbiome diversity in the bronchial tracts of patients with chronic obstructive pulmonary disease. J Clin Microbiol. 2012;50:3562-8.

36. Sze MA, Dimitriu PA, Hayashi S, et al. The lung tissue microbiome in chronic obstructive pulmonary disease. Am J Respir Crit Care Med. 2012; 185:1073-80.

37. Galiana A, Aguirre E, Rodriguez JC, et al. Sputum microbiota in moderate versus severe patients with COPD. Eur Respir J. 2014;43:1787-90.

38. Millares L, Ferrari R, Gallego M, et al. Bronchial microbiome of severe COPD patients colonised by Pseudomonas aeruginosa. Eur J Clin Microbiol Infect Dis. 2014;33(7):1101-11.

39. Garcia-Nuñez M, Millares L, Pomares X, et al. Severity-related changes of bronchial microbiome in chronic obstructive pulmonary disease. J Clin Microbiol. 2014;52:4217-23.

40. Sethi S. Infectious etiology of acute exacerbations of chronic bronchitis. Chest. 2000;117:380S-5S.

41. Huang YJ, Sethi S, Murphy T, et al. Airway microbiome dynamics in exacerbations of chronic obstructive pulmonary disease. J Clin Microbiol. 2014;52:2813-23.

42. Sethi S, Evans N, Grant BJ, Murphy TF. New strains of bacteria and exacerbations of chronic obstructive pulmonary disease. N Engl J Med. 2002;347:465-71.

43. Huang YJ, Kim E, Cox MJ, et al. A persistent and diverse airway microbiota present during chronic obstructive pulmonary disease exacerbations. Omics.. 2010;14:9-59.

44. Molyneaux PL, Mallia P, Cox MJ, et al. Outgrowth of the bacterial airway microbiome after rhinovirus exacerbation of chronic obstructive pulmonary disease. Am J Respir Crit Care Med. 2013;188:1224-31.

45. Sze MA, Dimitriu PA, Suzuki M, et al. Host Response to the Lung Microbiome in Chronic Obstructive Pulmonary Disease. Am J Respir Crit Care Med. 2015;192:438-45.

46. Sibley CD, Grinwis ME, Field TR, et al. Culture enriched molecular profiling of the cystic fibrosis airway microbiome. PLoS One. 2011;6:e22702.

47. Brown PS, Pope CE, Marsh RL, et al. Directly sampling the lung of a young child with cystic fibrosis reveals diverse microbiota. Ann AmThorac Soc. 2014;11:1049-55.

48. Renwick J, McNally P, John B, et al. The microbial community of the cystic fibrosis airway is disrupted in early life. PloS One. 2014;9:e109798.

49. Coburn B, Wang PW, Diaz Caballero J, et al. Lung microbiota across age and disease stage in cystic fibrosis. Sci Rep. 2015;5:10241. 
50. Bacci G, Paganin P, Lopez L, et al. Pyrosequencing Unveils Cystic Fibrosis Lung Microbiome Differences Associated with a Severe Lung Function Decline. PLoS One. 2016;11:e0156807.

51. Fodor AA, Klem ER, Gilpin DF, et al. The adult cystic fibrosis airway microbiota is stable over time and infection type, and highly resilient to antibiotic treatment of exacerbations. PloS One. 2012;7:e45001.

52. Carmody LA, Zhao J, Schloss PD, et al. Changes in cystic fibrosis airway microbiota at pulmonary exacerbation. Ann Am Thorac Soc. 2013;10:179-87.

53. Reid DW, Latham R, Lamont IL, et al. Molecular analysis of changes in Pseudomonas aeruginosa load during treatment of a pulmonary exacerbation in cystic fibrosis. J Cyst Fibros. 2013;12:688-99.

54. Lim YW, Evangelista JS 3rd, Schmieder R, et al. Clinical insights from metagenomic analysis of sputum samples from patients with cystic fibrosis. J Clin Microbiol. 2014;52:425-37.

55. Smith DJ, Badrick AC, Zakrzewski M, et al. Pyrosequencing reveals transient cystic fibrosis lung microbiome changes with intravenous antibiotics. Eur Respir J. 2014;44:922-30.

56. Gifford AH, Alexandru DM, Li Z, et al. Iron supplementation does not worsen respiratory health or alter the sputum microbiome in cystic fibrosis. J Cystic Fibros. 2014;13:311-8.

57. Smith DJ, Anderson GJ, Lamont IL, et al. Accurate assessment of systemic iron status in cystic fibrosis will avoid the hazards of inappropriate iron supplementation. J Cyst Fibros. 2013;12:303-4.

58. Bernarde C, Keravec M, Mounier J, et al. Impact of the CFTR-potentiator ivacaftor on airway microbiota in cystic fibrosis patients carrying a G551D mutation. PLoS One. 2015;10:e0124124.

59. Hauser PM, Bernard T, Greub G, et al. Microbiota present in cystic fibrosis lungs as revealed by whole genome sequencing. PloS One. 2014;9:e90934.

60. Quinn RA, Lim YW, Maughan H, et al. Biogeochemical forces shape the composition and physiology of polymicrobial communities in the cystic fibrosis lung. MBio. 2014;5:e00956-13.

61. Kim SH, Clark ST, Surendra A, et al. Global Analysis of the Fungal Microbiome in Cystic Fibrosis Patients Reveals Loss of Function of the Transcriptional Repressor Nrg1 as a Mechanism of Pathogen Adaptation. PLoS Pathog. 2015;11:e1005308.

62. McDonnell MJ, Jary HR, Perry A, et al. Non cystic fibrosis bronchiectasis: A longitudinal retrospective observational cohort study of Pseudomonas persistence and resistance. Respir Med. 2015;109:716-26.

63. Rogers GB, Zain NM, Bruce KD, et al. A novel microbiota stratification system predicts future exacerbations in bronchiectasis. Ann Am Thorac Soc. 2014;11:496-503.

64. Rogers GB, Bruce KD, Martin ML, et al. Corrections. The effect of long-term macrolide treatment on respiratory microbiota composition in non-cystic fibrosis bronchiectasis: an analysis from the randomised, double-blind, placebo-controlled BLESS trial. Lancet Respir Med. 2015;3:e15.

65. Rogers GB, Bruce KD, Martin ML, et al. The effect of long-term macrolide treatment on respiratory microbiota composition in non-cystic fibrosis bronchiectasis: an analysis from the randomised, double-blind, placebocontrolled BLESS trial. Lancet Respir Med. 2014;2:988-96.

66. Tunney MM, Einarsson GG, Wei L, et al. Lung microbiota and bacterial abundance in patients with bronchiectasis when clinically stable and during exacerbation. Am J Respir Crit Care Med. 2013;187:1118-26.

67. Huang YJ, Nelson CE, Brodie EL, et al. Airway microbiota and bronchial hyperresponsiveness in patients with suboptimally controlled asthma. J Allergy Clin Immunol. 2011;127:372-81 e1-3.

68. Marri PR, Stern DA, Wright AL, Billheimer D, Martinez FD. Asthma-associated differences in microbial composition of induced sputum. J Allergy Clin Immunol. 2013;131:346-52 e1-3.

69. Castro-Nallar E, Shen Y, Freishtat RJ, et al. Integrating microbial and host transcriptomics to characterize asthma-associated microbial communities. BMC Medical Genomics. 2015;8:50

70. Perez-Losada M, Castro-Nallar E, Bendall ML, et al. Dual Transcriptomic Profiling of Host and Microbiota during Health and Disease in Pediatric Asthma. PLoS One. 2015;10:e0131819.

71. Huang YJ, Nariya S, Harris JM, et al. The airway microbiome in patients with severe asthma: Associations with disease features and severity. J Allergy Clin Immunol. 2015;136:874-84.

72. Zhang Q, Cox M, Liang Z, et al. Airway Microbiota in Severe Asthma and Relationship to Asthma Severity and Phenotypes. PLoS One. 2016;11:e0152724.

73. Liu AH. Revisiting the hygiene hypothesis for allergy and asthma. J Aller gy Clin Immunol. 2015;136:860-5.

74. Webley WC, Aldridge KL. Infectious asthma triggers: time to revise the hygiene hypothesis? Trends Microbiol. 2015;23:389-91.

75. Ege MJ, Mayer M, Normand AC, et al. Exposure to environmental microorganisms and childhood asthma. N Engl J Med. 2011;364:701-9.

76. Stein MM, Hrusch CL, Gozdz J, et al. Innate Immunity and Asthma Risk in Amish and Hutterite Farm Children. N Engl J Med. 2016;375:411-21.

77. Han MK, Zhou Y, Murray S, et al. Lung microbiome and disease progression in idiopathic pulmonary fibrosis: an analysis of the COMET study. Lancet Respir Med. 2014;2:548-56.

78. Molyneaux PL, Cox MJ, Willis-Owen SA, et al. The role of bacteria in the pathogenesis and progression of idiopathic pulmonary fibrosis. Am J Respir Crit Care Med. 2014;190:906-13.

79. Nakano Y, Yang IV, Walts AD, et al. MUC5B Promoter Variant rs35705950 Affects MUC5B Expression in the Distal Airways in Idiopathic Pulmonary Fibrosis. Am J Respir Crit Care Med. 2016;193:464-6.

80. Zhu QQ, Zhang XL, Zhang SM, et al. Association Between the MUC5B Promoter Polymorphism rs35705950 and Idiopathic Pulmonary Fibrosis: A Meta-analysis and Trial Sequential Analysis in Caucasian and Asian Populations. Medicine (Baltimore). 2015;94:e1901.

81. The Lancet Respiratory Medicine. The changing landscape of idiopathic pulmonary fibrosis. Lancet Respir Med. 2014;2:507.

82. Faner R SO, Agustí A, Bernasconi E, et al. The Microbiome in Respiratory Medicine: Current Challenges and Future Perspectives. Eur Resp J. 2017. 